\title{
Interacting with sonification systems: closing the loop
}

\author{
Andy Hunt, Thomas Hermann, Sandra Pauletto \\ University of York, UK, Bielefeld University, Germany, University of York, UK \\ \{adh@ohm.york.ac.uk, thermann@techfak.uni-bielefeld.de, sp148@ohm.york.ac.uk \}
}

\begin{abstract}
This paper stresses the importance of the human user being tightly embedded within an interactive control loop for exploring data sets using sound. We consider the quality of interaction, and how this can be improved in computer systems by learning from real-world acoustic interactions. We describe how different sonification methods can utilise the human feedback loop to enhance the perception and analysis of the data under investigation. Some considerations are given regarding systems and applications.
\end{abstract}

\section{Introduction}

This paper discusses the way that humans interact with sound in everyday life. It focuses on how we gain feedback from a combination of senses, helping us to obtain our sense of reality, and thus to understand the world better. We consider the nature of 'Control Intimacy'; the quality of interaction that we take for granted in manipulating everyday objects, but which is so often lacking in limited-interaction, visual-only interfaces.

Musical instruments are examined as a particular type of human-device interface (tried and tested over long periods of time) which allow such control intimacy to develop to high levels. We then consider the special case of computer interaction where the human is allowed to form intimate control loops with the system, using sound (and other senses) as feedback.

We outline what can be learnt about integrating interaction into exploratory data analysis techniques. We then consider some challenging application areas which can be tackled in a fresh way specifically using continuous interaction with sonic feedback.

\section{Control Loops in Human Interaction}

As human beings, from the moment we are born we begin to interact with the world. In fact a baby's first action in the world is to cry - to make a sound. As we grow we learn first how to control our bodies, and then how to interact with objects around us. The way that the world works - its physical laws, the constants and the variables - becomes coded into our developing brain. We learn to take for granted that dropped objects fall to the ground, and that when we reach for an object we feel it and see it and hear it as we touch it. Watch a young child playing with a pile of bricks and you will notice how she develops her movements by interacting with objects and obtaining from them instant and continuous feedback of their position, speed and texture.

Such control loops of human action and continuous feedback from the world become embedded deep within our mind-body system.

Therefore it is hardly surprising that, later in life, we become rapidly frustrated with computer systems that engage with us in a very different and more limited manner [1]. Here, too often, the interaction is dictated by the computer. A prompt is given, or a list of options presented as icons or a menu. We have to choose from the selection offered by the computer at every stage of the process, and thus the interaction becomes a series of stilted prompt-choice cycles; a far cry from the way that we have learnt to interact with the everyday world. It is as if we have designed our computer systems to always remain outside our control loop. We seem to expect them always to be under 'third-party' control; things to which we give instructions. The result of this is that we rarely gain the same intimacy of control with a computer as we do with objects in everyday life. A common observation is that much of our time working with computers is spent in navigating the interface, rather than completing the task.

It matters too whether or not you are part of the control loop. Many passengers become travel sick whereas this condition rarely affects drivers. When you are controlling an object you know what to expect, as - by definition - you are initiating the reactions and can thus prepare your mental apparatus for the result. Maybe you have had the experience of being in a room where someone else is in charge of the TV remote control. You cannot believe how much they are "playing around with it', driving to distraction everyone else in the room. However when you have it, everything is different, and you are 'simply seeing what's on the next channel'. It matters greatly whether you are in the control loop or not.

This paper considers how we can bring more real-world interaction into our computing interfaces, by placing the human operator firmly in charge of a continuous control loop wherever possible.

\subsection{Control intimacy}

A child playing with wooden blocks and a person operating a typical computer interface are both interacting with external objects. It is just that the quality of the interaction is different. The extent to which the interaction directly affects the object is one 
aspect of the control intimacy being exhibited; the other aspect being how well the human manages this control. Real-world objects seem to exhort us to spend time with them, and as we do, we subconsciously learn more about them, and master the skills of manipulating them until the control becomes almost automatic.

We are all aware of situations where we are controlling an object and almost forget that we are doing it. Car drivers often report that they are shocked to find themselves at their destination, without knowing how they got there; even though the act of driving is an extremely complex interactive process. Many good performing musicians feel that their fingers are somehow playing the music by themselves. In musical performances, their minds appear to be concentrating on higher-level modes of expression, whilst their bodies are managing the physical act of manipulating the instrument. In fact, most musicians will recount the terrifying feeling of suddenly becoming aware of what their fingers are doing, and as a result the performance grinds to a halt!

Csikszentmihalyi [2] called this type of disembodied interaction flow. He explains how it is found freely in children as they play, and less so in adult life. Certainly in most computer interfaces the flow is never allowed to happen, due to the constant choices, and the stop-start style of the interaction caused by the emphasis on reading words, processing and selecting options. To shed some light on how to improve this state of affairs, let us consider the special case of interaction where the goal is to generate sound.

\subsection{The special case of interacting with sound}

Engineers use sound to deduce the internal state of engines and complex machinery such as washing machines. Sound warns us of dangers outside our relatively narrow field of view. It is also the medium by which much human communication takes place via speech and singing.

Whenever we interact with a physical object, sound is made. It confirms our initial contact with the object, but also tells us about its properties; whether it is solid or hollow, what material it is made of etc. The sound synchronises with both our visual and tactile 'views' of the object. As we move the object, the sounds it makes give us continuous feedback about its state. Sound is a temporal indicator of the physical state of the world around us.

The act of making sound may be satisfying to human beings precisely because they are in a very tightlyresponsive control loop. This does not by definition mean that other people find the sound satisfying. Think of times when a person mindlessly 'drums' his fingers on the table to help him think. He is part of the control loop, and so is expecting the moment-by-moment sonic response. The whole process often remains at the subconscious level, and he is unaware he is doing it. However, to other people in the vicinity (not in the loop) the sound can be intensely annoying. Therefore, we see that there is something special about being the one to initiate actions, and receive constant and immediate sonic results.

\subsection{Tuning parameters for individuals}

An observation about the individuality of interacting with sound became clear to the first author during his experiences as an amateur radio operator. It is wellknown to 'Radio Hams' that there is quite an art to 'tuning in' the radio to pick out a particularly weak signal. Somehow you need to be able to pick out the signal you are trying to listen to, in spite of the fact that there are much louder interfering signals nearby in the frequency spectrum, and background noise, and all manner of fluctuating signal levels and characteristics due to propagation conditions. To do this requires a fine balance with the tuning control, and the signal modulation controls, and sometimes even movement of the antenna. When two people are listening to the same radio signal, but only one is at the controls, it is quite common for the signal to be audible only to the person at the controls.

What can we learn from such an observation? Perhaps when a sound is made by a system, we ought to consider who the sound is intended for. Is it just for the person 'in the loop', since s/he is the one controlling the system parameters? Or, is the sound intended for everyone? Where data is being portrayed as sound, for example in a hospital environment, it is important that everyone recognises the sound. However, where the sound is being controlled interactively by a person, we might need to be aware that the operator could be inadvertently tuning the system for themselves. More complex sounds (which could appear as annoying or unpleasant) can be quite acceptable to people who are in the control loop.

The more general point to be inferred from the above example is that humans can use physical interaction to control the generation and modulation of sound in order to extract data from a noisy signal. Section 3 studies this area in more detail.

Musical instruments are a special case of sound generating device where the main intention is that other people do indeed listen to the sound. Having said that, if you are sharing a house with someone practicing an instrument (particularly if the player is a beginner), the observation that 'it-matters-whether-you-are-in-controlloop' becomes obvious.

In the next section we look at human interaction with instruments in more detail.

\subsection{Musical instruments as exemplars}

The sonic response of physical objects is so deeply ingrained in the human psyche that sound and music has been a fundamental part of every known human society. In this section, we take a closer look at human interaction with musical instruments; since much can be learned from this about what makes good quality real-time interaction.

In a previous paper [3] we described the attributes of most acoustic musical instruments as follows:

\section{- $\quad$ there is interaction with a physical object.}


- co-ordinated hand and finger motions are crucial to the acoustic output.

- $\quad$ the acoustic reaction is instantaneous.

- the sound depends in complex ways on the detailed kinds of interaction (e.g. on simultaneous positions, velocities, accelerations, and pressures).

The physical interaction with the instrument causes an instantaneous acoustic reaction. This allows the player to utilise the everyday object manipulation skills he has developed all his life. The player's energy is directly responsible for activating the sonic response of the system; when the player stops, the sound dies away. The mapping of system input to sonic output [4] is complex; many input parameters are cross-coupled, and connected in a non-linear manner to the sonic parameters. This can make an instrument difficult to play at first, but offers much scope for increased subtlety of control over time. As the player practices, he becomes better and better. This allows the control intimacy to increase to a level where the physical operation of the instrument becomes automatic. At this point the player often experiences the 'flow' of thinking at levels much higher than complex physical interface manipulations.

We should also not underestimate the importance of tactile feedback. A good performer will rarely look at her instrument, but will instead rely on the years of training, and the continuous feel of the instrument which is tightly coupled to the sound being produced. The human operator learns to wrap his mind-body system around the instrument to form a human-machine entity.

So, it seems from considering how people interact with musical instruments, that devices intended for exploration need to have certain characteristics. These include a real-time sonic response, a complex control mapping which permits learning, and tactile feedback tightly coupled to the sonic response.

\section{The use of sound in exploratory data analysis}

In this section we consider the use of sound in computers as a way of understanding data taken from the world around us. We describe how sound can be used to portray data, and explain the importance of continuous human interaction with the sound generating process.

\subsection{Sonification}

The general term Auditory Displays is employed to describe the use of sound in computers to portray information. It covers a wide range of topics including alarm signals, earcons and sonification techniques, most of which are discussed by the International Community for Auditory Display (ICAD) [5]. Sonification is the more specific term used to describe the rendering of data sets as sound, or:

“. . the transformation of data relations into perceived relations in an acoustic signal for the purposes of facilitating communication or interpretation" [6]

Humans must be equipped with several senses for a good reason: that they are complementary, and are needed in collaboration to gain a full sense of the world around us. There are several areas where sound offers improvements over visual feedback.

"The main differences of sound displays over visual displays are that sound can:

- $\quad$ represent frequency responses in an instant (as timbral characteristics)

- $\quad$ represent changes over time, naturally

- $\quad$ allow microstructure to be perceived

- $\quad$ rapidly portray large amounts of data

- $\quad$ alert the listener to events outside the current visual focus

- $\quad$ holistically bring together many channels of information" [7]

So, the use of sound allows us to gain alternative insights into the data under examination. Until recently the sheer computing power required to generate the sound output has meant that, by necessity, the act of sonification was a non-interactive process. Data was loaded, parameters were selected, the algorithm set going, and some time later the sound emerged. Too often in computing technology, when this time-lag is eliminated by improvements in processor speed, the style of interaction remains; and interaction is limited to setting parameters, then listening to a completed sound. As stated in section 2 , this stilted interaction prevents any form of control intimacy from developing. In the following section we examine how to re-introduce interaction into the art of making sound.

\subsection{Interacting with Sonification}

Now that computers can run fast enough to generate sound in real-time, we should re-design our data-tosound algorithms to take advantage of the rich possibilities of continuous human interaction. How are we to allow a 'flow' experience of data sonification to take place?

This question was examined by the 2004 Interactive Sonification workshop, organised by the first two authors [7] and is summarised in [8]. At this gathering researchers from diverse disciplines described the magic that occurs when sound is generated in real-time under human control. Although musical instruments also generate sound in real-time under human control, their primary is artistic expression. In contrast, the goal of an interactive sonification system is to allow humans to explore and understand the intrinsic properties of a particular data set. In other words, it is an analysis tool. In sections 4 and 5, we describe toolkits that we have developed which enable such interaction to be explored, and some interactive sonification applications which are in progress at the time of writing. There are two basic approaches to the incorporation of interaction into a sonification algorithm. The first involves taking data attributes and converting them into sound (so called 
parameter mapping), whilst allowing the user to interact with this process. The second involves designing a sonification model which is inherently interactive.

Where data is time-ordered (for example where it has been gathered from a time-evolving source) it is sensible to retain this time order by mapping the data onto sound variables. Traditionally, the entire data set is converted into a sound file, which is then listened to noninteractively, rather like a CD. However, interaction can be built into the process to allow a human being to explore the data much more freely. For example the position in the data can be moved continuously, 'scrubbing' through the data and instantly hearing the sonic result. Alternatively the data could play back continuously in a loop while the sonification algorithm is tuned by the user, rather analogous to the Radio Ham example given in section 2.3.

The next section describes how sonification models can be designed and used for exploring non-time-based data sets.

\subsection{Using model-based sonification to enhance user interaction}

Traditional sonification schemes are based on clearly separated computation and playback phases, as pointed out above. In contrast, the framework of Model-based sonification (MBS) involves "interacting with datadriven virtual acoustic objects" - which is by design inherently interactive. This approach is almost orthogonal to previous techniques: whereas in parameter mapping sonification the data is used to provide controls (e.g. playing instructions) for a given instrument (sound synthesis algorithm), in Model-based sonification the data is used to establish the instrument or algorithm itself. This means that with an MBS system the user is given the responsibility of interacting with the sonification model, and (only) by this means causes the sonification to generate sound. MBS is thus different from parameter mapping sonification, in that there is no mapping from data to model-parameters - instead the data become (in most models) part of the model configuration and thus do not explicitly but implicitly determine the sonification.

Model-based sonification is a concept in which a virtual acoustic object is established, dependent on the data under analysis. It thus provides a method of mediating between abstract data spaces and the infinite space of possible instruments. Concrete models usually specify the laws of dynamics that govern the temporal evolution of the dynamical elements constituting the 'virtual instrument'. Typically sonification models are set up first to be in a state of equilibrium so that they do not produce any sound without being excited into a nonequilibrium state. Most MBS models are dissipative (because, for example, sound radiation represents energy loss) which causes the sound to vanish after interaction ceases.

Model design is a very creative process. Some example models have been described in previous work, [9][10][11], but the best suited models for specific analysis tasks are still to be invented. Model design entails wide possibilities for bringing task-oriented needs into the concrete realisation of a model. Complex sound responses can occur, but humans respond well to this type of reaction. The MBS concept and its benefits are discussed in detail in [9].

In this paper we focus on the aspects of engagement and flow, which have been shown to play an important role in the use of interactive auditory systems such as musical instruments.

The following three aspects of acoustic real-world interactions cause human users to increase their engagement with the system:

(i) sound complexity,

(ii) low-latency correspondence to human controls, (iii) attention.

Concerning $(i)$, the complexity of sounds from realworld acoustic systems is much higher than that of most sounds used in computer systems. This is because realworld systems typically possess complex dynamic behaviour involving nonlinearities as well as stochastic components, whereas synthesised sounds are often generated by rather 'sterile' algorithms such as FMsynthesis. Our auditory system is so well tuned to, and experienced with, analysing real-world sounds that it appreciates complexity, often interpreting this as 'beauty' of sound. In contrast, even complex stochastic time series generated in computer contexts (e.g. from chaotic systems) fail to please or convince the listener. For instance plucking a guitar string will never lead to the exactly same sound, whereas sonification systems typically reproduce sound accurate to the single bit. Model-based sonification provides exactly this 'mind of its own' to a data-driven dynamical acoustic system. Since high-quality interactions (those that go far beyond a simple triggering) are unique excitation patterns, the resulting sound will also be a unique reaction to this unrepeatable stimulus. Sound complexity is not granted automatically by the use of an MBS approach. Instead we need to learn from real-world acoustics, which provides inspiration on suitable ways to create complex sonic dynamics, resulting in sounds where users can rely on their highly developed listening skills.

Concerning (ii), low-latency is an important factor in creating engagement and for facilitating the user's transition from conscious mode to flow mode. Lowlatency sound generation is useful for guiding exploratory activities since the immediate response allows the user to directly refine his control activities. It is also important for increasing the synchronisation of other modalities occurring in the interaction, such as tactile and visual feedback. For example the user hears the resulting sound at the same time as they experience the tactile feedback from the control device.

Concerning (iii), attention; users often focus their attention in order to enhance perception. Think for instance of a photograph you are looking at, wondering why you took a picture of a boring landscape. Later you remember that you were taking a photo of a bird. It is almost invisible on the picture, but your attention 
'magnified' at the time. Attention is the magnifying lens through which users experience and explore the world! Attention is often directed towards correlations between the user's activities and a system's response to it. Even faint correlations can then receive significant magnification by attention, but only for the user in the control loop. Attention is tightly coupled to points $(i)$ and (ii). Complexity of sound grants the availability of many possible sources of correlations between sound and the system feedback. Low-latency is an important factor for ensuring that these correlations are easily detectable in the interaction. Attention is thus related to a user's engagement with the system, since the occurrence of structure on multiple complexity levels keeps alive the user's interest in practising and improving in the interaction.

Model-based sonification helps to implement these aspects automatically since it incorporates an interaction style which is more like real-world acoustic interactions. However, the designer has the freedom to refine the sonification so that the aspects mentioned above come better into play. For instance:

- by allowing non-linear couplings of the dynamical elements, so that the sonification model exhibits a rich acoustic behaviour. This may be computationally costly but the evolution of computation power makes it merely a matter of time.

- by enhancing the modes of interaction. For instance a sonification model triggered by a computer mouse is 'poorer' than one in which users bring in the multi-dimensional controls of a whole articulated hand, which in turn is poorer than interactions with tangible interfaces that take the user even closer to real-world acoustic interaction.

- by designing sonification models so that subtle changes of the excitation pattern (e.g. of position or velocity) are directly related to subtle changes of the sound. As an example think of a sonification model with which the user can interact by clicking on a graphical representation of the data points. One possible paradigm of model excitation would be to give the entire excitation to the nearest data node. In this case any click within the vicinity of the node will cause the same sonification. If, however, the excitation energy is distributed between the nearest neighbours according to their distance, then subtle changes in the activation position will result in subtle changes in the sound.

In this way, we hope that the above aspects prove helpful in the design process of engaging exploratory sonification models.

\section{Software for interactive sonic data analysis}

Sonification systems which allow us to link data sets to their acoustic representation face several requirements in terms of interfaces, structure, and performance. Here we briefly introduce interactive sonification toolkits (ISTs). We then step back and regard the general requirements for software being used in sonification systems.
At the University of York we have been developing an Interactive Sonification Toolkit [12] which allows rapid prototyping of the transition from data to sound, coupled with real-time user interaction. It is constructed in PD [13] so that the end product is also cross-platform and open-source. $\mathrm{Pd}$ allows real-time sound synthesis, creation of graphical user interfaces, refinement of the 'program' during runtime, easy interfacing with many sorts of sensors/controllers, e.g. via MIDI or OSC, and platform independency.

This is similar in concept to an interactive sonification platform produced in Bielefeld, based on a graphical simulation system Neo/NST [14], which is particularly strong in data computation, data mining, data visualisation, and rapid prototyping. However, it is weaker in real-time sound synthesis and limited to the Linux platform. All sonification models mentioned above have been implemented using Neo/NST, using Neo displays for graphical data representation and interaction.

Interactive sonification systems have to consist of several components, which (a) need tight interaction, (b) are computationally expensive, or (c) demand special platforms. These are expanded below.

a) All sonification systems involve data-related computations. Interaction with the display (such as selection) requires intermediate representations to be recomputed. This demands a tight connection between the controls and the data computation engine. Neo/NST here provides a good platform, and related alternatives for powerful data processing are MatLab or Octave.

b) Specifically for sonification models, CPU power is never enough. It is useful to distribute specialised rendering routines onto an extra machine. Where appropriate, sonification models can be divided into a high-level part (where low-level synthesis instructions are computed) and a low-level sound engine (where the sound signal is actually generated). For the first part, simulation systems such as Neo/NST or languages like Smalltalk are appropriate. For sound computation, there are several candidates, e.g. Csound, PD, or Supercollider. c) The third aspect concerns controls for sonification models. Many suitable interfaces, such as an audiohaptic ball interface [15] or computer-vision-based gestural interfaces are works-in-progress and demand their own machine. Other interfaces (e.g. certain joysticks, Phantom device, etc.) are only supported on special platforms/OS and do not allow a tight direct combination.

In summary, it seems that any isolated platform is so far inappropriate for solving the whole range of problems encountered in interactive multimodal displays. Many different aspects need to come together in order to enable a quick and effortless design of systems. Heterogeneous solutions not only offer the chance to distribute the computation better over several machines, but also to use optimised components according to the respective needs. An intelligent architecture for such complex systems is currently under development at Bielefeld University and will be presented elsewhere. 


\section{Applications}

In this section we briefly outline projects that are in the early stages of development.

\subsection{Analysis of time-stamped data}

At present the PD-based toolkit described above is being modified to suit several different projects. Two of these are funded by EPSRC, allowing advanced data mining of helicopter flight data and physiotherapy muscle data respectively. The data produced by a helicopter test flight cannot be adequately shown on a computer screen at a reasonable resolution whilst giving an overall picture; so we are using sonification to allow engineers to navigate the entire data set in a matter of seconds. Physiotherapists wish to know more about the qualitative aspects of the signals produced by the movement of muscles, and sound has allowed new insights above and beyond the traditional visual plots [12].

\subsection{Landmine detection}

The number of anti-personnel landmines buried around the world is estimated to be between 50 and 70 million. Their impact on third world countries is devastating in terms of local economies and their impact on the local population. Humanitarian landmine detection and clearance is currently a slow process, because of the high false alarm rate associated with current detector technology

Sponsored by the UK Department for International Development, ERA Technology have developed a prototype hand-held detector which uses both groundpenetrating radar (GPR) and metal detection (MD) to significantly reduce the effect of false alarms and also detect minimum metal (plastic) landmines.

The key factors in the design of the new detector are affordability and ease of use. ERA have developed an audio interface which uses frequencies in the $100 \mathrm{~Hz}$ to $3 \mathrm{kHz}$ range to give continuous feedback to the user of the GPR detector. The depth of the target is given by the frequency of the output signal, and the size of the target is given by the amplitude of the signal.

"A key feature of the design is a special (patented) man-machine acoustic interface. This approach utilises the inherent capabilities of humans to 'process' information and keeps the 'man-in-the-loop'.' [16]

The metal detector also produces its own audio tone, and together the operator has sonic feedback of the objects on and below the surface.

The University of York, Department of Electronics, are planning to work with ERA to investigate ways of combining the separate signals from the two sensors, and to optimise the presentation of the audio information for users from many cultural backgrounds.

\section{Conclusions}

In this paper we have stressed the importance, for complex data analysis, of the human user being in a tightly-coupled intimate control loop. We have illustrated this with examples from everyday interaction, particularly with sound. We have also summarised the work done in building interaction into sonification techniques, and the inherently interactive method of model-based sonification.

In conclusion, the research community needs to be acutely aware of the quality of interaction that is provided in human interfaces, in order to maximise the capabilities of the human mind-body system.

\section{Acknowledgements}

Thanks to the Sensors Design Consultancy team of ERA Technology for the input and collaboration regarding their existing work on audio interfaces for landmine detection. Thanks to EPSRC for funding the work described in sections 4 and 5, under the project 'Improved data mining through an interactive sonic approach'.

\section{References}

[1] A. Hunt, Radical User Interfaces for Real-time Musical Control, Ph.D. thesis, University of York, 2000, http://www-users.york.ac.uk/ elec18/download/adh_thesis

[2] M. Csikszentmihalyi, Beyond Boredom and Anxiety: Experiencing Flow in Work and Play, reprint, Jossey Bass Wiley, 2000.

[3] Hermann, T. \& Hunt, A.D, The discipline of Interactive Sonification, Proc. 1st Int. Workshop on Interactive Sonification, Bielefeld, Germany, January 2004. http://www.interactive-sonification.org

[4] A. D. Hunt, M. Paradis, and M. Wanderley, "The importance of parameter mapping in electronic instrument design," Journal of New Music Research, vol. 32, no. 4, pp. 429-440, December 2003, special issue on New Musical Performance and Interaction.

[5] International Community for Auditory Display http://www.icad.org/

[6] Gregory Kramer et al., Sonification Report, National Science Foundation, 1997 http://www.icad.org/websiteV2.0/References/nsf.html

[7] http://www.interactive-sonification.org

[8] Andy Hunt and Thomas Hermann, "The importance of interaction in sonification," in Proceedings of the Int. Conf. on Auditory Display. ICAD, 2004, submitted.

[9] Thomas Hermann, PhD, Sonification for Exploratory Data Analysis, Bielefeld University, 2002

[10] Hermann, T., Meinicke, P., \& Ritter, H. Principal Curve Sonification, Proc. Int. Conf. on Auditory Display p8186, 2000

[11] Hermann, T.\& Ritter, H. Crystallization Sonification of High-dimensional Datasets, Proc. Int. Conf. on Auditory Display p76--81, 2002

[12] Pauletto, S \& Hunt, A., "An interactive sonification toolkit" in Proceedings of the Int. Conf. on Auditory Display. ICAD, 2004.

[13] www.pure-data.org

[14] H. Ritter, The Graphical Simulation Toolkit Neo/NST http://www.techfak.unibielefeld.de/ags/ni/projects/neo/neo_e.html

[15] Hermann, T., Krause, j., \& Ritter, H. Real-Time Control of Sonification Models with an Audio-Haptic Interface, Proc. Int. Conf. on Auditory Display p82-86, 2002

[16] http://www.era.co.uk/docs/electronics/Minetect.pdf 